\title{
Geração Automática de Modelos Dinâmicos de Cabos
}

\author{
Automatic Generation of Cable's Dynamic Models \\ Thiago Avila Pouzada e Sebastião Cícero Pinheiro Gomes
}

Universidade Federal do Rio Grande, Rio Grande, RS, Brasil

thiago.pouzada@gmail.com; sebastiaogomes@furg.br

\section{Resumo}

Modelos dinâmicos de cabos constituem um tema de pesquisa de grande importância atualmente, principalmente em razão de existirem muitas aplicações práticas subaquáticas correlatas à indústria do petróleo. Entretanto, devido às não linearidades e à necessidade de se trabalhar com muitos graus de liberdade, desenvolver modelos dinâmicos para cabos é uma tarefa difícil. Neste artigo é proposto um formalismo discreto, o qual supõe que o cabo é formado por vários elos rígidos conectados por articulações elásticas fictícias. Considerou-se uma das extremidades do cabo articulada a uma estrutura flutuante (plataforma ou navio) e, na outra extremidade, consideraram-se dois casos: carga terminal livre; extremidade fixa ao fundo do mar. Os cabos estão submersos e considerou-se um modelo simples para o arrasto hidrodinâmico, proporcional ao quadrado da velocidade relativa entre o fluido e a estrutura. Utilizando o formalismo de Euler-Lagrange, foram desenvolvidos, analiticamente, modelos considerando-se dois, três e quatro elos. A partir desses modelos foram identificados padrões de crescimento dos seus vetores e matrizes, os quais possibilitaram a proposição de algoritmos genéricos que permitem a geração automática dos modelos, para um número qualquer de elos, representando de forma discreta a flexibilidade contínua do cabo. Simulações foram realizadas e mostraram qualitativamente resultados conforme o esperado fisicamente. Um software foi desenvolvido para mostrar animações das configurações espaciais do cabo obtidas a partir de simulações numéricas, animações estas que mostraram uma grande sensação de realidade física.

Palavras-chave: Estrutura flexível, cabo, modelagem dinâmica, formalismo discreto, aplicações subaquáticas.

\section{Abstract}

Dynamic modeling of cables is a research theme of great importance and practical application nowadays in the underwater oil industry. However, due to non-linearity behavior present on cable dynamics, as well as the need to work with many degrees of freedom, to develop dynamic models for these systems becomes a hard task. To develop dynamic models of cables, in this article is proposed a discrete formalism, which supposes the cable consisting of rigid links connected by fictitious elastic joints. The cables were considered with one of its endings articulated to a floating structure (ship or platform), while on the other ending were considered two cases: one free terminal loading; ending fixed to the ground. The cables are submersed and the hydrodynamic drag was considered from a simple model, proportional to the square of the relative velocity between the structure and the fluid. Using the Euler-Lagrange formalism, the dynamic models for cables considering two, three and four links were developed. From these models algorithms have been developed to automatically generate dynamic models for any number of links to represent in a discrete form the cable's continuous flexibility. Simulations were performed, which showed the results agreeing qualitatively with the physical expected. A software has been developed to display animation from the spatial configuration of the cable. This animation, based on data from numerical simulation, allowed us show that the developed dynamic models give a great sense of physical reality.

Keywords: Flexible structures, cable, dynamic modeling, discrete formalism, underwater applications. 


\section{Introdução}

O desenvolvimento de modelos dinâmicos para estruturas flexíveis do tipo cabo constitui atualmente um tema relevante em razão de diversas aplicações, voltadas principalmente para a indústria do petróleo em explorações subaquáticas. Cabos umbilicais de veículos subaquáticos do tipo ROV (Remotely Operated Vehicle), cabos de amarração ou ancoragem, risers e cabos para reboque de embarcações são exemplos de estruturas utilizadas em uma planta de exploração subaquática de petróleo. Estas estruturas são complexas por envolverem dinâmicas com muitos graus de liberdade e, portanto, apresentam, naturalmente, grande dificuldade no desenvolvimento dos seus modelos dinâmicos. O importante interesse industrial, bem como os desafios científicos correlatos ao tema, motivaram o desenvolvimento da pesquisa cujos resultados estão resumidos no presente artigo, que contém uma proposta original de algoritmos para a determinação automática de modelos dinâmicos de estruturas flexíveis do tipo cabo.

A maior parte dos artigos científicos sobre a modelagem de cabos utiliza a técnica de elementos finitos, cujo objetivo final geralmente consiste na determinação das forças em equilíbrio estático, importantes para o dimensionamento do cabo. Recentemente, Bamdad (2013) propôs uma solução analítica para a dinâmica de manipuladores suspensos por cabos. Bi et al. (2013) aplica o Método dos Elementos Finitos para estudar as tensões presentes em cabos umbilicais submarinos. Chang et al. (2008) investiga o comportamento dinâmico de um cabo com base na teoria catenária. Chatjigeorgiou e Mavrakos (2010) propõem uma solução para a modelagem dinâmica de cabos utilizando a técnica das diferenças finitas. Escalante et al. (2011) utiliza o Método dos Elementos Finitos aplicado a cabos para a obtenção de um modelo de ordem reduzida, a partir de decomposições ortogonais. Fang et al. (2012) representa a flexão e efeitos de torção para cabos de reboque, também usando o Método dos Elementos Finitos. Gobat e Grosenbaugh (2001) analisam as deficiências na integração do modelo de um cabo, quando se utiliza o método de diferenças finitas. Guo et al. (2007) investiga o controle de vibração induzida pelo vento em uma única torre de uma ponte estaiada com um amortecedor de massa pêndulo multi-estágio (MSPMD), utilizando o Método dos Elementos Finitos. Liu et al. (2013) estuda características dinâmicas de cabos em linhas de transmissão, com base na formulação Lagrangeana. Luongo et al. (2008) utiliza métodos de soluções, tais como diferenças finitas e o Método de Galerkin, para estudar a dinâmica de cabos suspensos. Masciola et al. (2012) estuda um método de solução para a configuração estática de um cabo, a partir de uma aproximação discreta. Nahon (1999) desenvolve um modelo para uma antena de rádio telescópio, baseado em um formalismo discreto (lumped mass). Pathote (2014) usa a curva $b$-spline em uma nova técnica de modelagem matemática para cabos tensionados. Pereira (2010) investiga a interação fluido-estrutura de um cabo submerso, a partir do acoplamento da dinâmica do cabo com o movimento do fluido, utilizando uma aproximação discreta e o formalismo Lagrangeano para a dinâmica do cabo (esta tese de doutorado foi publicada de forma compactada em (Pereira et al., 2012). Seo et al. (2010) apresenta uma análise dinâmica (utilizando o Método dos Elementos Finitos) simplificada de uma ponte suspensa, sujeita a movimentos causados por eventos sísmicos. Srivastava et al. (2011) apresenta uma abordagem numérica por diferenças finitas, a fim de prever o comportamento dinâmico de cabos utilizados em reboque de embarcações, quando o navio de reboque tem sua velocidade linearmente alterada. Vaz e Patel (1995) apresentam uma solução numérica para o movimento transiente de cabos marítimos utilizados em reboques. Wolfschluckner e Jodin (2013) comparam o Método dos Elementos Finitos com métodos numéricos e analíticos, para descrever o comportamento dinâmico de um cabo. Zanela (2013) usa uma abordagem discreta para a modelagem tridimensional de estruturas flexíveis do tipo cabo.

No presente trabalho foram consideradas duas situações diferentes: o cabo articulado a uma plataforma móvel numa das suas extremidades e livre na outra; o cabo articulado à plataforma móvel numa das suas extremidades e fixo ao fundo do mar na outra. A plataforma móvel (que pode ser um navio) foi considerada com a possibilidade de mover-se somente na direção vertical, simulando as oscilações que ocorrem na superfície do mar. O formalismo de Euler-Lagrange foi usado para obter o modelo dinâmico do cabo, cuja estrutura flexível foi aproximada por uma equivalente discreta, formada por elos rígidos conectados por articulações elásticas fictícias. Modelos foram desenvolvidos analiticamente para os casos do cabo aproximado por dois, três e quatro elos. Em Pouzada e Gomes (2015) encontram-se os resultados para o caso do cabo aproximado por quatro elos. As equações crescem muito com o acréscimo do número de elos, de forma que se torna completamente inviável o desenvolvimento analítico manual das equações, para além de quatro elos. Porém, com o desenvolvimento dos modelos até quatro elos, foi possível detectar a existência de padrões de crescimento para os vetores e matrizes do modelo dinâmico. Uma vez obtidos esses padrões, algoritmos genéricos foram desenvolvidos para gerar automaticamente as equações para os modelos dinâmicos de cabos, considerando-se um número qualquer de elos para aproximar de forma discreta a flexibilidade contínua. O presente artigo completa de forma definitiva o trabalho de Pouzada e Gomes (2015), uma vez que os 
algoritmos genéricos aqui propostos possibilitam o desenvolvimento de modelos dinâmicos considerando-se um número grande de elos. Um software que permite realizar animações foi desenvolvido especialmente para a análise dos resultados de simulações, cujo principal objetivo é testar qualitativamente se o cabo comporta-se conforme o esperado fisicamente.

\section{Modelagem dinâmica}

Os cabos considerados na pesquisa que originou esse artigo têm uma das extremidades articulada a uma plataforma ou embarcação na superfície do mar e, na outra extremidade, consideraram-se dois casos: carga terminal de massa $m_{c}$ livre; cabo fixo ao fundo do mar. Em ambos os casos, supõe-se que os movimentos do cabo ocorrem em um único plano. Muitas aplicações práticas permitem aproximações planas, tais como cabos de ancoragem, de amarrações, risers, etc., conforme ilustrado na Figura 1. Considera-se ainda que o movimento da extremidade do cabo que é fixa à plataforma flutuante acontece apenas na direção vertical.

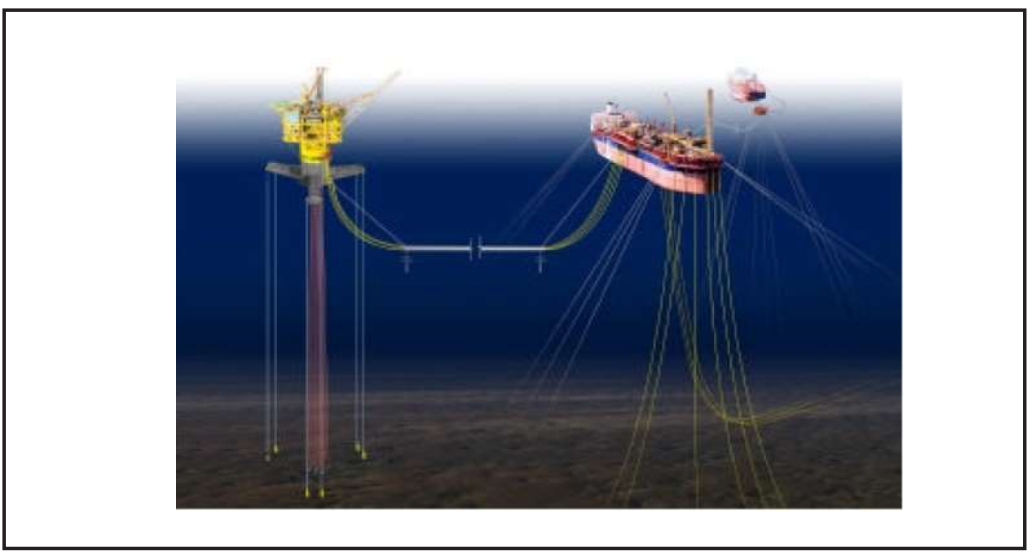

Figura 1: Cabos em aplicações subaquáticas (fonte: http://diariodopresal.wordpress.com/petroleo-e-gas).

Foi utilizado um formalismo discreto, supondo o cabo dividido em diversos elos rígidos conectados por articulações elásticas fictícias, conforme ilustrado na Figura 2. Supõe-se ainda que cada articulação fictícia possui a sua constante elástica e o seu coeficiente de atrito conhecidos.

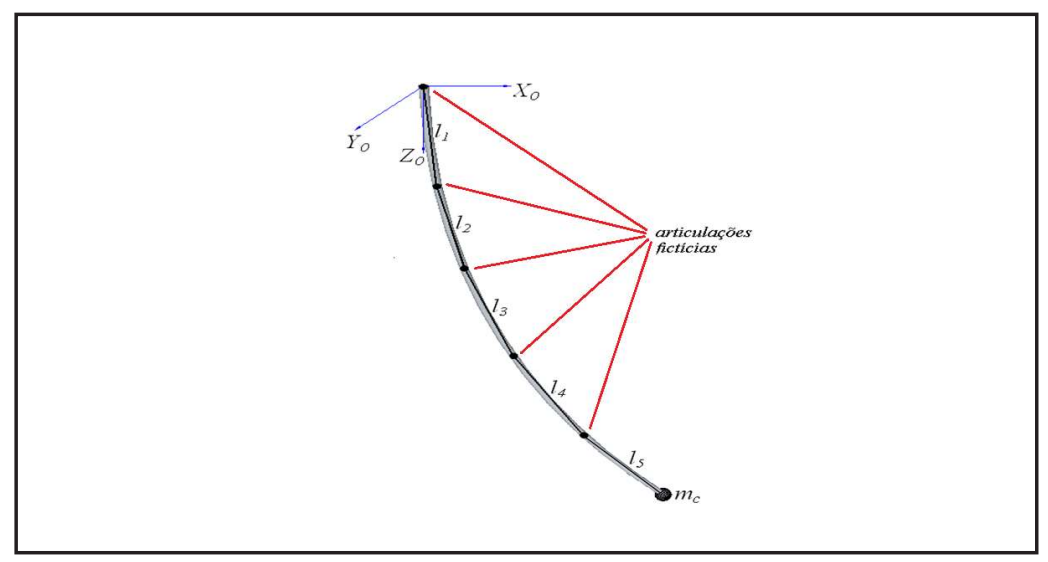

Figura 2: Aproximação discreta para a flexibilidade contínua do cabo.

Modelos dinâmicos considerando-se dois, três e quatro elos foram desenvolvidos analiticamente. A Figura 3 mostra uma ilustração do cabo dividido em três elos. O empuxo cancela o peso no caso da plataforma flutuante e a dinâmica imposta pelo movimento da superfície do mar foi aproximada por um sistema massa-mola- amortecedor. 


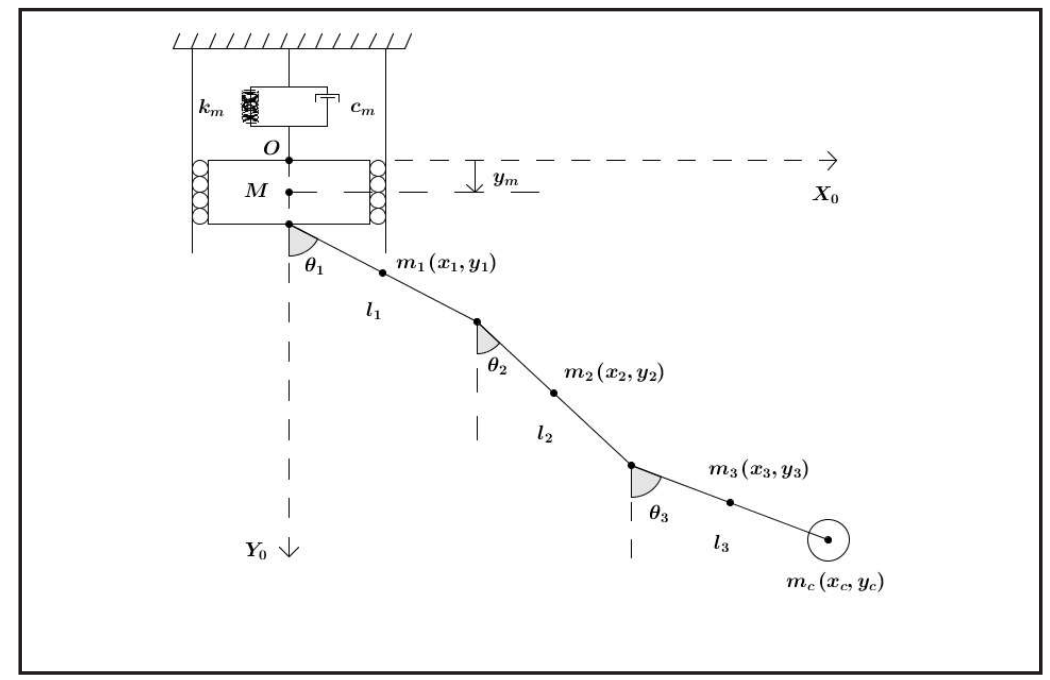

Figura 3: Formalismo discreto utilizando-se três elos.

O vetor com as coordenadas de posição para a estrutura com três elos possui a forma:

$$
\vec{y}=\left[\begin{array}{llll}
y_{m} & \theta_{1} & \theta_{2} & \theta_{3}
\end{array}\right]^{T}
$$

A dinâmica com $n$ elos tem $n+1$ graus de liberdade. $y_{m}$ é a posição vertical da plataforma, medida com relação à origem do referencial inercial, situada na superfície média do mar. $\theta_{i}, i=1, \ldots, n$, são os ângulos de elevação, medidos com relação à direção vertical.

A energia cinética de translação pode ser escrita na forma:

$$
E_{C_{T_{i}}}=\frac{1}{2} m_{i}\left(\dot{x}_{i}^{2}+\dot{y}_{i}^{2}\right)
$$

onde: $m_{i}$ são as massas de cada elo; $x_{i}$ e $y_{i}$ são as posições na horizontal e na vertical, respectivamente, dos centros de massa de cada elo, medidas com relação ao referencial inercial com origem na superfície média do mar, com $i=1, \ldots, n$.

A energia cinética de rotação é devida aos movimentos angulares em torno dos centros de massa dos elos, sobre eixos perpendiculares aos elos e passando por seus centros de massa:

$$
E_{C_{R_{i}}}=\frac{m_{i}}{12} l_{i}^{2}+\dot{\theta}_{i}^{2}
$$

onde $l_{i}$ são os comprimentos de cada elo.

A energia cinética total da estrutura é escrita na forma:

$$
E_{C}=\frac{1}{2} M \dot{y}_{m}^{2}+\sum_{i=1}^{n}\left(E_{C_{T_{i}}}+E_{C_{R_{i}}}\right)
$$

A energia potencial devida à elasticidade em cada articulação possui a forma:

$$
E_{P_{E_{i}}}=\frac{1}{2} k_{i}\left(\theta_{i+1}-\theta_{i}\right)^{2}, \quad i=1, \ldots, n-1
$$

onde $k_{i}$ são as constantes elásticas em cada articulação.

A energia potencial gravitacional em cada elo é escrita conforme a seguinte equação: 


$$
E_{P_{G_{i}}}=m_{i} g h_{i}
$$

onde: $h_{i}$ correspondem às alturas dos centros de massa de cada elo, relativas às posições destes quando a estrutura encontra-se em repouso e na posição vertical; $g$ é a aceleração gravitacional na superfície da Terra.

A energia potencial total da estrutura é escrita na forma:

$$
E_{P}=\frac{1}{2} k_{m} y_{m}^{2}+m_{c} g h_{c}+\sum_{i=1}^{n-1} E_{P_{E_{i}}}+\sum_{i=1}^{n} E_{P_{G_{i}}}
$$

onde: $m_{c}$ e $h_{c}$ correspondem à massa e à altura da carga terminal, respectivamente, quando esta é livre; $k_{m}$ é a constante elástica relativa ao movimento vertical da plataforma flutuante ou embarcação.

Uma vez determinadas as energias cinética e potencial da estrutura, o seu Lagrangeano é facilmente obtido:

$$
L=E_{C}-E_{P}
$$

Aplicando-se as equações de Euler-Lagrange aos $n+1$ graus de liberdade, o modelo dinâmico do sistema é obtido e pode ser escrito na forma:

$$
I(\vec{y}) \ddot{\vec{y}}+C \dot{\vec{y}}+K \vec{y}+\vec{F}(\vec{y}, \dot{\vec{y}})+G(\vec{y})=\vec{T}_{m}
$$

$I(\vec{y})$ é a matriz de inércia, $C$ é a matriz de coeficientes de atrito, $K$ é a matriz de constantes elásticas e $\vec{F}(\vec{y}, \dot{\vec{y}})$ o vetor de esforços Coriolis-centrífugos. $G(\vec{y})$ é o vetor de esforços gravitacionais, no qual estão inclusos os esforços devidos ao empuxo atuante nos elos e na carga terminal. As forças de empuxo são consideradas atuando nos centros de massa de cada corpo rígido da cadeia poli-articulada e ainda na mesma direção da força gravitacional, mas com sentido oposto. $\vec{T}_{m}$ é o vetor com esforços externos, no qual estão inclusos os esforços devidos ao arrasto hidrodinâmico. O fluido (água) foi considerado em repouso (ausência de correntes subaquáticas) e em cada elo a força de arrasto atua no seu centro de massa e é proporcional ao quadrado da sua velocidade linear, na forma:

$$
\alpha_{i}=-\gamma_{i} v_{i}^{2} \operatorname{sign}\left(v_{i}\right)=-\gamma_{i} \frac{l_{i}^{2}}{4} \dot{\theta}_{i}^{2} \operatorname{sign}\left(\dot{\theta}_{i}\right)
$$

onde: $\gamma_{i}$ é o coeficiente de arrasto do elo $i$ e $v_{i}$ a sua velocidade linear.

\section{Algoritmos genéricos}

O desenvolvimento analítico das equações dos modelos dinâmicos do cabo, para os casos de se considerar dois, três e quatro elos, permitiu a identificação dos padrões de crescimento dos vetores e matrizes dos modelos. Uma vez identificados, os padrões de crescimento permitiram a obtenção de algoritmos genéricos, capazes de gerar de forma automática os modelos dinâmicos, para um número qualquer de elos considerados na aproximação discreta da flexibilidade contínua do cabo. Uma vez implementados, esses algoritmos possibilitaram a realização de diversas simulações que permitiram verificar uma boa sensação de realidade física, conforme será demonstrado posteriormente.

A matriz de inércia é simétrica e de ordem $n+1$, de forma que o seu algoritmo de geração (Figura 4) determina apenas os elementos da diagonal principal e os acima desta. $M$ é a massa da plataforma flutuante, enquanto $l_{i}$ e $m_{i}$, com $i=1, \ldots, n$, correspondem, respectivamente, aos comprimentos e massas de cada um dos elos. $m_{\mathcal{c}}$ é a massa da carga terminal.

A matriz de coeficientes de atrito $C$ é simétrica e de ordem $n+1$, sendo $c_{m}$ o coeficiente de atrito relativo ao movimento vertical da estrutura flutuante, enquanto $c_{i}$, com $i=1, \ldots, n$, correspondem aos coeficientes de atrito em cada articulação fictícia (conforme a Figura 5). A matriz de constantes elásticas obedece à mesma regra de formação da matriz de coeficientes de atrito e assim, o mesmo algoritmo pode ser usado, apenas trocando-se $C$ por $K, c_{m}$ por $k_{m}$ e $c_{i}$ por $k_{i}$ no algoritmo apresentado como Figura 5. 


$$
\begin{aligned}
& \text { for } \begin{array}{l}
i=1: n+1, \\
\text { for } j=1: n+1, \\
\text { if } i=1, \\
\quad \text { if } j=i, \\
\quad I(i, j)=M+m_{c}+\sum_{k=1}^{n} m_{k} ;
\end{array}
\end{aligned}
$$

end if,

$$
\text { if } j>i \text {, }
$$

$$
I(i, j)=-\left(\frac{m_{j-1}}{2}+m_{c}+\sum_{k=1}^{n} m_{k}\right) l_{j-1} \sin \left(\theta_{j-1}\right) ;
$$

end if,

else

$$
\begin{aligned}
& \text { if } j=i, \\
& \qquad I(i, j)=\left(\frac{m_{i-1}}{4}+m_{c}+\sum_{k=1}^{n} m_{k}\right) l_{i-1}^{2}+I_{r i}
\end{aligned}
$$

end if,

if $j>i$,

$$
I(i, j)=\left(\frac{m_{i}}{2}+m_{c}+\sum_{k=1}^{n} m_{k}\right) l_{i-1} l_{j-1} \cos \left(\theta_{i-1}-\theta_{j-1}\right) ;
$$

\section{end if,}

end if,

$$
I(j, i)=I(i, j)
$$

end,

end, 


$$
\begin{aligned}
& \text { for } i=1: n, \\
& \qquad \begin{array}{l}
\text { for } j=1: n, \\
\quad a(i, j)=0 ; \\
\quad \text { if } i=j \text { and } i<n, \\
\quad a(i, j)=c_{i}+c_{i+1} ;
\end{array}
\end{aligned}
$$

end if,

if $i=j$ and $i=n$,

$$
a(i, j)=c_{i}
$$

end if,

if $j=i+1$,

$$
\begin{aligned}
& a(i, j)=-c_{j} ; \\
& a(j, i)=a(i, j) ;
\end{aligned}
$$

end if,

end,

end,

for $i=1: n+1$,

for $j=1: n+1$,

$C(i, j)=0$

if $i=1$ and $j=1$,

$$
C(i, j)=c_{m}
$$

end if,

if $i>1$ and $j>1$,

$$
C(i, j)=a(i-1, j-1) ;
$$

end if,

end,

end,

Figura 5: Algoritmo de geração da matriz de coeficientes de atrito. 
Para o vetor Coriolis-centrífugo, o primeiro elemento tem geração diferente dos demais, conforme mostra o primeiro termo da Figura 6. Os outros elementos $\left(f_{2}, \ldots, f_{n+1}\right)$ são obtidos conforme o algoritmo da mesma figura. Nesta equação, $\beta$ é um parâmetro que depende dos valores de $i$ e $j$, conforme visto na Figura 7.

No caso do vetor de esforços gravitacionais, seu primeiro elemento é nulo, conforme mostra o primeiro termo da Figura 8. Este primeiro elemento está relacionado à plataforma flutuante e é nulo porque o seu peso é anulado com o seu empuxo. Os outros elementos do vetor gravitacional são determinados a partir do algoritmo na Figura 8.

\section{Simulações}

As simulações foram realizadas considerando-se os dois casos previamente citados: uma das extremidades articulada à plataforma flutuante e com a outra extremidade livre (carga terminal de massa $m_{c}$ ); articulado à plataforma em uma das extremidades e com a outra extremidade fixa ao fundo do mar. O modelo dinâmico é o mesmo. A diferença é que, no segundo caso, adota-se uma carga terminal com massa muito grande $\left(m_{c}=8 \times 10^{8} \mathrm{~kg}\right)$ e ainda, o seu empuxo é igual ao seu peso. Os parâmetros adotados para a realização das simulações foram os seguintes:

$l=240 m$ (Comprimento do cabo);

$l_{i}=\frac{l}{n}$ (Comprimento de cada elo);

$r_{i}=0.01 \mathrm{~m}$ (Diâmetro dos elos);

$\rho_{c}=7850 \frac{\mathrm{kg}}{\mathrm{m}^{3}}$ (Massa específica do cabo);

$m_{c}=60 \mathrm{~kg}$ (Massa da carga terminal);

$m_{c}=8 \times 10^{8} \mathrm{~kg}$ (Massa da carga terminal, quando o cabo é fixo ao fundo do mar);

$M=6000 \mathrm{~kg}$ (Massa da plataforma flutuante);

$c_{m}=237.6 \frac{\mathrm{Ns}}{\mathrm{m}}$ (Coeficiente de atrito relativo ao movimento vertical da plataforma);

$c_{i}=2.8 \frac{\mathrm{Ns}}{\mathrm{rd}}$ (Coeficiente de atrito em cada articulação fictícia);

$k_{i}=104.7 \frac{\mathrm{N}}{\mathrm{rd}}$ (Constante elástica em cada articulação fictícia);

$k_{m}=1200 \frac{\mathrm{N}}{m}$ (Constante elástica relativa ao movimento vertical da plataforma);

$\gamma_{i}=200 \frac{N s^{2}}{(r d . m)^{2}}$ (Coeficiente de arrasto hidrodinâmico).

As simulações são mostradas a seguir na forma de uma sequência de frames, relativos à configuração espacial do cabo em cada instante de tempo diferente. Foram considerados 60 e 80 elos para representar a flexibilidade contínua $(n=60$ e $n=80)$.

A primeira simulação foi realizada com o cabo aproximado por 60 elos e em queda livre a partir de uma determinada configuração espacial inicial. O cabo está sujeito ao empuxo e ao arrasto hidrodinâmico. A Figura 9 ilustra o movimento do cabo nesta situação, cujo tempo total foi de $31 \mathrm{~s}$ e os frames mostrados a cada $1 \mathrm{~s}$.

A segunda simulação consiste no cabo com 60 elos e fixo ao fundo do mar. O cabo também está sujeito ao arrasto hidrodinâmico e ao empuxo. Foi aplicada uma força vertical na plataforma flutuante equivalente a:

$$
u(t)=25000 \sin (0.5 \pi t) N
$$

A estrutura inicialmente está em repouso. A Figura 10 ilustra o movimento do cabo nesta simulação, com um tempo total de $15.5 \mathrm{~s}$ e frames mostrados a cada $0.5 \mathrm{~s}$.

\section{Conclusões}

Os modelos dinâmicos desenvolvidos mostraram resultados de simulações fisicamente esperados em ambos os casos, ou seja, queda livre e cabo com extremidade final fixa ao fundo do mar. Este último caso é semelhante a cabos de ancoragem ou amarração usados em plataformas ou navios, os quais constituem importantes aplicações tecnológicas no ambiente subaquático. Os algoritmos genéricos propostos permitem gerar os modelos dinâmicos de forma automática, considerando-se um número qualquer de elos para representar a flexibilidade contínua do cabo, sendo esta a maior contribuição do presente artigo. Foi desenvolvido um software para a animação gráfica dos resultados de simulações e este permitiu mostrar uma grande sensação de realidade física nos movimentos do cabo, em todas as simulações realizadas. As próximas fases da presente pesquisa consistirão em expandir o formalismo de modelagem para movimentos em três dimensões e incluir cargas terminais com dinâmica própria, como um ROV, por exemplo. 


$$
\begin{aligned}
& f_{1}=-\sum_{k=1}^{n}\left[\left(\frac{m_{k}}{2}+m_{c}+\sum_{g=k+1}^{n} m_{g}\right) l_{k} \cos \left(\theta_{k}\right) \dot{\theta}_{k}^{2}\right] \\
& \text { for } i=1: n \text {, } \\
& \qquad f_{i+1}=l_{i} \sum_{\substack{j=1 \\
j \neq i}}^{n}\left[\beta l_{j}\left(\begin{array}{c}
\frac{m_{\max (i, j)}}{2}+m_{c}+ \\
+\sum_{g=\max (i, j)+1}^{n} m_{g}
\end{array}\right)\left(\begin{array}{c}
-\frac{1}{2} \operatorname{sign}(\beta) \cos \left(\theta_{i}\right) \sin \left(\theta_{j}\right)+ \\
+\frac{1}{\beta} \sin \left(\theta_{i}\right) \cos \left(\theta_{j}\right)
\end{array}\right) \dot{\theta}_{j}^{2}\right] ; \\
& \text { end, }
\end{aligned}
$$

Figura 6: Elementos do vetor Coriolis-centrífugo.

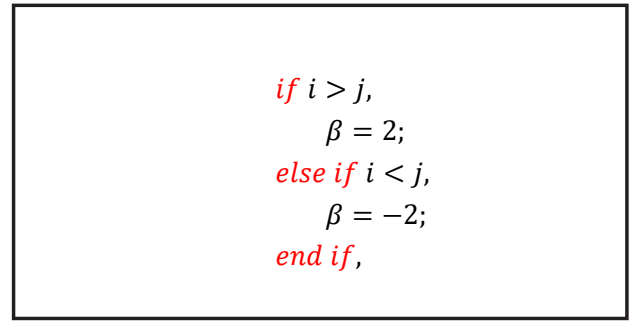

Figura 7: Parâmetro $\beta$.

$$
\begin{aligned}
& G(1)=0 \\
& \text { for } i=2: n+1 \\
& \qquad G(i)=\left(\frac{m_{i-1}}{2}+m_{c}+\sum_{k=i}^{n} m_{k}\right) g l_{i-1} \sin \left(\theta_{i-1}\right)
\end{aligned}
$$

Figura 8: Elementos do vetor de esforços gravitacionais. 

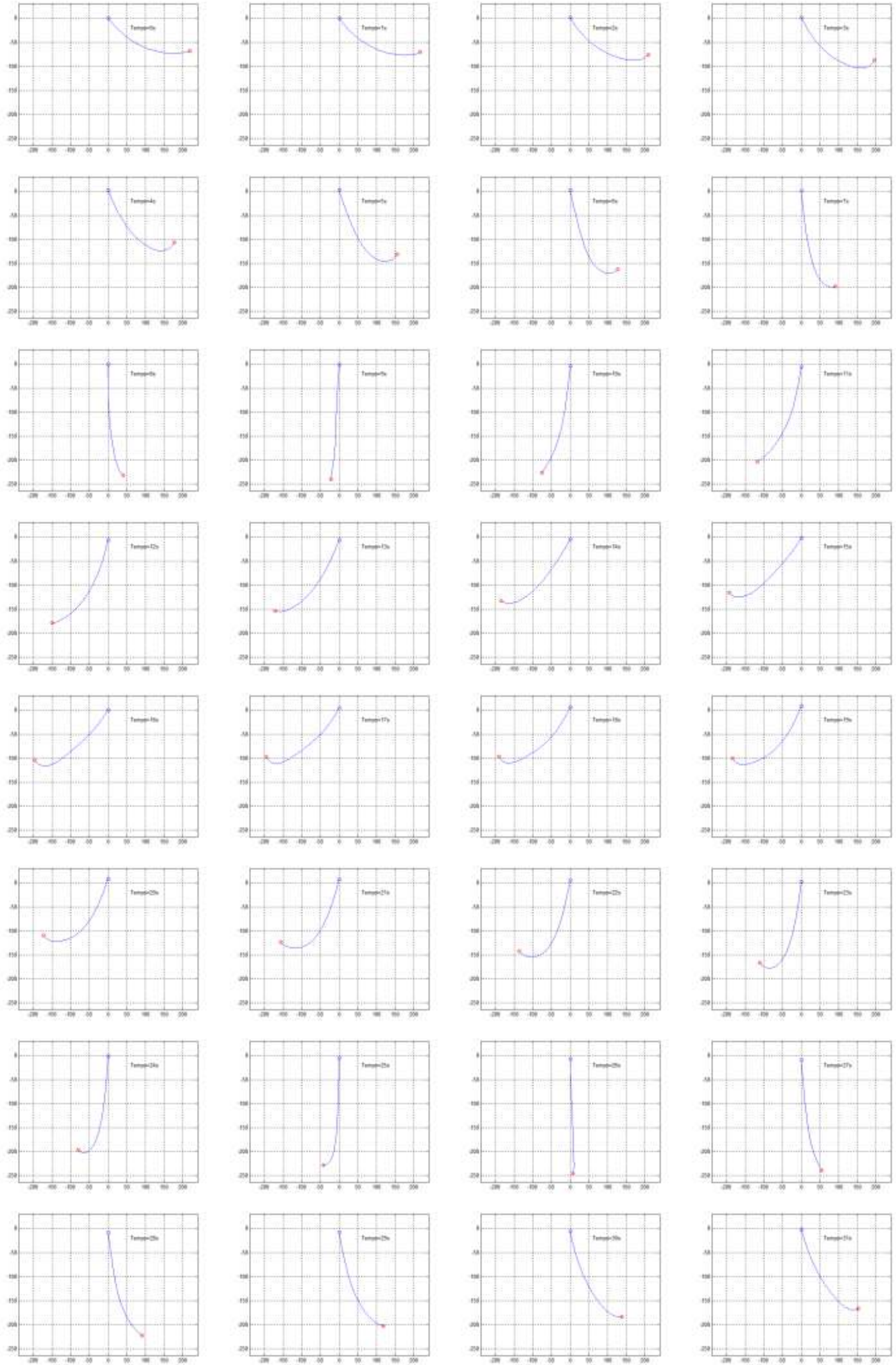

Figura 9: Cabo aproximado por 60 elos, em queda livre, 31s de tempo total de simulação, com frames a cada 1 s. 

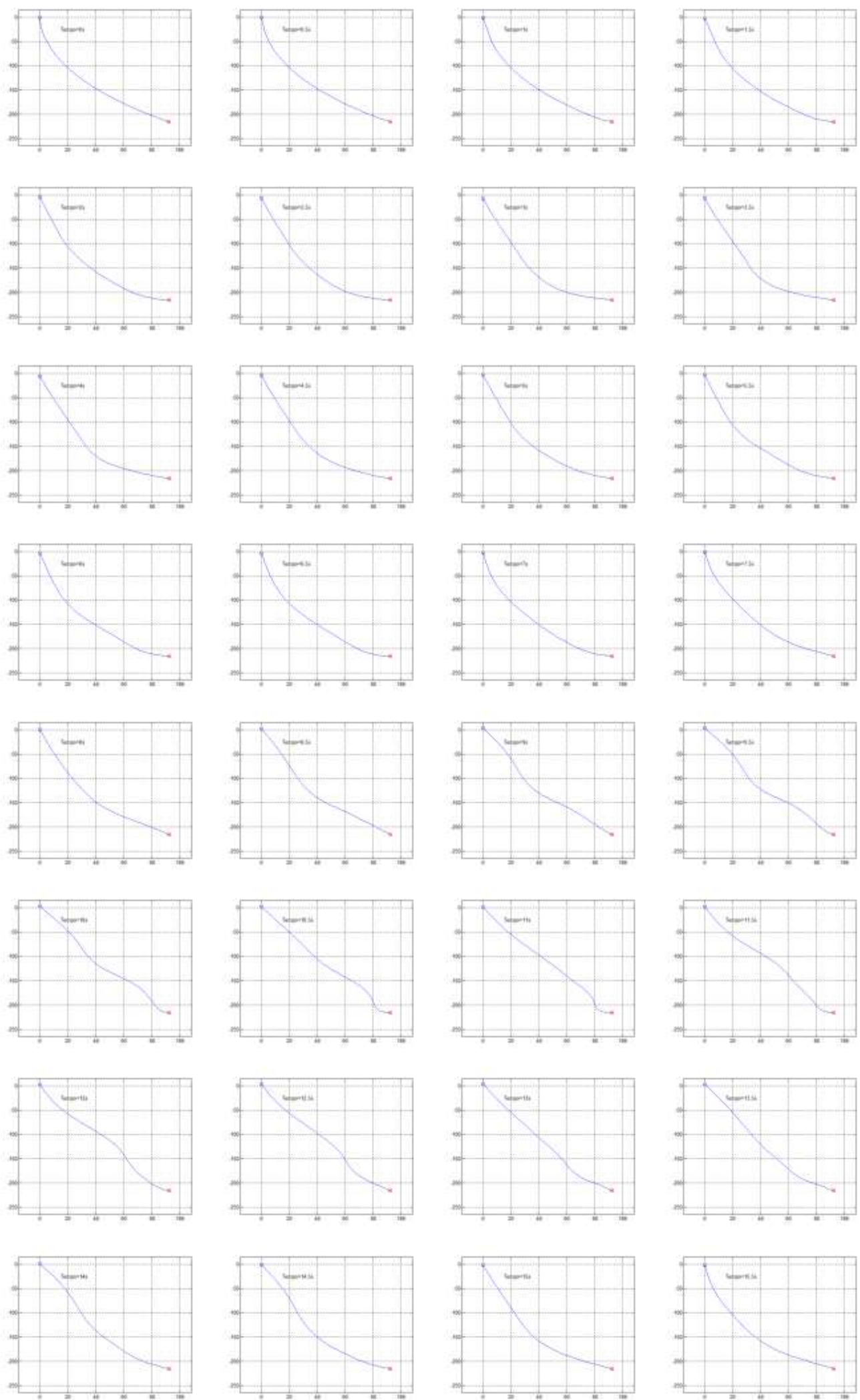

Figura 10: Cabo aproximado por 60 elos e fixo ao fundo do mar, com 15.5s de tempo total de simulação e frames a cada $0.5 \mathrm{~s}$. 


\section{Referências}

Bamdad, M. (2013). Analytical dynamic solution of a flexible cable-suspended manipulator. Front Mech Eng, 8(4), 350-359.

Bi, G., Zhu, S., Liu, J., Fang, X., Wang, L. (2013). Dynamic simulation and tension compensation research on subsea umbilical cable laying system. J Marine Sci Appl, 12, 452-458.

Chang, S., Park, J., Lee, K. (2008). Nonlinear dynamic analysis of spatially suspended elastic catenary cable with finite element method. KSCE Journal of Civil Engineering, 12(2), 121-128.

Chatjigeorgiou, I. K., Mavrakos, S. A. (2010). The 3d nonlinear dynamics of catenary slender structures for marine applications. Nonlinear Dynamics Croatia: INTECH, p 366.

Escalante, M. R., Sampaio, R., Rosales, M. B., Ritto, T. (2011). A reduced order model of a 3d cable using proper orthogonal decomposition. Mecánica Computacional, XXX, 1143-1158.

Fang, Z., He, Q., Xiang, B., Xiao, H., He, K., Du, Y. (2012). A finite element cable model and its applications based on the cubic spline curve. China Ocean Eng, 27(5), 683-692.

Gobat, J. I., Grosenbaugh, M. A. (2001). Application of the generalized-alpha method to the time integration of the cable dynamics equations. Computer Methods in Applied Mechanics and Engineering, 190(37-38), 4817-4829.

Guo, A., Xu, Y., Li, H. (2007). Dynamic performance of cable-stayed bridge tower with multi-stage pendulum mass damper under wind excitations - i: Theory. Earthquake Engineering and Engineering Vibration, 6(3), 295-306.

Liu, Y., Qian, Z., Xia, K. (2013). Mechanical response of transmission lines based on sliding cable element. J Cent South Univ, 21, 3370-3377.

Luongo, A., Zulli, D., Piccardo, G. (2008). Analytical and numerical approaches to nonlinear galloping of internallyresonant suspended cables. Journal of Sound and Vibration, Elsevier, 315(3), 375-393.

Masciola, M. D., Nahon, M., Driscoll, F. R. (2012). Static analysis of the lumped mass cable model using a shooting algorithm. J Waterway, Port, Coastal, Ocean Eng, 138(2), 164-171.

Nahon, M. (1999). Dynamics and control of a novel radio telescope antenna. The American Institute of Aeronautics and Astronautics, AIAA, 99(4120), 214-222.

Pathote, P. (2014). Efficient mathematical model for prestressing cables and its implementation in computer analysis. IOSR Journal of Mathematics (IOSR-JM), 10(3-V), 32-38.

Pereira, A. E. L. (2010). O método da decomposição de adomian aplicado à interação fluido-estrutura de um cabo. Tese de Doutorado, Universidade Federal do Rio Grande do Sul (UFRGS).

Pereira, A. E. L., Gomes, S. C. P., de Bortoli, A. L. (2012). A new formalism for the dynamic modelling of cables. Mathematical and Computer Modelling of Dynamical Systems, 1, 1-14.

Pouzada, T. A., Gomes, S. C. P. (2015). Modelagem dinâmica de cabos para aplicações subaquáticas. Scientia Plena, 11, $1-10$.

Seo, Y., Jeong, U. Y., Cho, J. S. (2010). Simplified finite element dynamic analysis of cable-supported structures subjected to seismic motions. KSCE Journal of Civil Engineering, 14(4), 579-587.

Srivastava, V. K., Yvss, S., Tamsir, M. (2011). Dynamic behavior of underwater towed-cable in linear profile. International Journal of Scientific \& Engineering Research, 2(7).

Vaz, M. A., Patel, M. H. (1995). Transient behaviour of towed marine cables in two dimensions. Applied Ocean Research, Elsevier, 17, 143-153.

Wolfschluckner, A., Jodin, D. (2013). Modelling cable dynamics exemplified by load transposition of insulator strings in overhead lines. Proceedings in Manufacturing Systems, 8(3).

Zanela, E. B. (2013). Modelagem analítica de estruturas do tipo cabo para aplicações subaquáticas. Dissertação de Mestrado, Universidade Federal do Rio Grande (FURG). 